\title{
Existence and Asymptotic Stability of Solutions of a Functional Integral Equation via a Consequence of Sadovskii's Theorem
}

\author{
Agnieszka Chlebowicz, ${ }^{1}$ Mohamed Abdalla Darwish,, ${ }^{2,3}$ and Kishin Sadarangani ${ }^{4}$ \\ ${ }^{1}$ Department of Mathematics, Rzeszów University of Technology, al. Powstańców Warszawy 8, 35-959 Rzeszów, Poland \\ ${ }^{2}$ Department of Mathematics, Sciences Faculty for Girls, King Abdulaziz University, Jeddah 21589, Saudi Arabia \\ ${ }^{3}$ Department of Mathematics, Faculty of Science, Damanhour University, Damanhour 22511, Egypt \\ ${ }^{4}$ Departamento de Matemáticas, Universidad de Las Palmas de Gran Canaria, Campus deTafira Baja, \\ 35017 Las Palmas de Gran Canaria, Spain \\ Correspondence should be addressed to Agnieszka Chlebowicz; agnchleb@prz.edu.pl
}

Received 3 May 2014; Accepted 12 June 2014; Published 16 July 2014

Academic Editor: Józef Banaś

Copyright (c) 2014 Agnieszka Chlebowicz et al. This is an open access article distributed under the Creative Commons Attribution License, which permits unrestricted use, distribution, and reproduction in any medium, provided the original work is properly cited.

Using the technique of measures of noncompactness and, in particular, a consequence of Sadovskii's fixed point theorem, we prove a theorem about the existence and asymptotic stability of solutions of a functional integral equation. Moreover, in order to illustrate our results, we include one example and compare our results with those obtained in other papers appearing in the literature.

\section{Introduction}

Measures of noncompactness play very important role in nonlinear analysis. They are often applied to the theories of differential and integral equations as well as to the operator theory and geometry of Banach spaces. The concept of a measure of noncompactness was initiated by Kuratowski [1] and Darbo [2]. In [2] Darbo, by using the concept of a measure of noncompactness, proved a fixed point theorem. In [3] Sadovskii improved the results obtained in [2].

The purpose of this paper is to present a theorem on the existence and asymptotic stability of solutions of a functional integral equation. Our study will be placed in the Banach space of real functions which are defined, continuous, and bounded on the real half-axis $\mathbb{R}_{+}$. The functional integral equation studied in the paper contains as particular cases a lot of functional and integral equations appearing in the literature. The main tool used in our investigations is a consequence of Sadovskii's fixed point theorem [3].

\section{Notations, Definitions, and Auxiliary Facts}

Let $E$ be a given real Banach space with a norm $\|\cdot\|$. For a nonempty subset $X$ of $E$ denote by $\bar{X}$ the closure of $X$ and by Conv $X$ the closed convex hull of $X$. For $X$ and $Y$ being subsets of $E$, by $X+Y$ and $\lambda X, \lambda \in \mathbb{R}$, we denote the usual algebraic operations on $X$ and $Y$. Further, let $\mathfrak{M}_{E}$ denote the family of all nonempty and bounded subsets of $E$ and $\mathfrak{N}_{E}$ its subfamily consisting of all relatively compact subsets. If $\gamma$ is a mapping defined on $\mathfrak{M}_{E}$ with real values, then by ker $\gamma$ we denote the following family:

$$
\operatorname{ker} \gamma=\left\{X \in \mathfrak{M}_{E}: \gamma(X)=0\right\} \text {. }
$$

This family will be called the kernel of the mapping $\gamma$. Following [4], we consider the following definition of the concept of a measure of noncompactness.

Definition 1. A mapping $\mu: \mathfrak{M}_{E} \rightarrow \mathbb{R}_{+}=[0, \infty)$ will be called a measure of noncompactness in $E$ if it satisfies the following conditions.

(1) The family $\operatorname{ker} \mu$ is nonempty and $\operatorname{ker} \mu \subset \mathfrak{N}_{E}$.

(2) $X \subset Y \Rightarrow \mu(X) \leq \mu(Y)$.

(3) $\mu(\bar{X})=\mu(X)$.

(4) $\mu(\operatorname{Conv} X)=\mu(X)$.

(5) $\mu(\lambda X+(1-\lambda) Y) \leq \lambda \mu(X)+(1-\lambda) \mu(Y)$ for $\lambda \in[0,1]$. 
(6) If $\left(X_{n}\right)$ is a sequence of closed subsets of $\mathfrak{M}_{E}$ such that $X_{n+1} \subset X_{n}$ and $\lim _{n \rightarrow \infty} \mu\left(X_{n}\right)=0$, then $\bigcap_{n=1}^{\infty} X_{n} \neq \phi$.

In [2] Darbo proved the following fixed point theorem.

Theorem 2. Let $\Omega$ be a nonempty, bounded, closed, and convex subset of $E$ and let $\mathscr{H}: \Omega \rightarrow \Omega$ be a continuous mapping such that there exists a constant $k \in[0,1)$ satisfying

$$
\mu(\mathscr{H} X) \leq k \mu(X)
$$

for any nonempty subset $X$ of $\Omega$, where $\mu$ is a measure of noncompactness.

Then $\mathscr{H}$ has a fixed point in $\Omega$.

In [3], Sadovskii proved the following generalization of Theorem 2.

Theorem 3. Let $\Omega$ be a nonempty, bounded, closed, and convex subset of $E$ and let $\mathscr{H}: \Omega \rightarrow \Omega$ be a continuous mapping such that

$$
\mu(\mathscr{H} X)<\mu(X)
$$

for any nonempty and noncompact subset $X$ of $\Omega$, where $\mu$ is a measure of noncompactness in E. Then $\mathscr{H}$ has a fixed point in $\Omega$.

Notice that in [3] Theorem 3 is proved for a particular measure of noncompactness in $E$, but the same argument serves for an arbitrary measure of noncompactness in $E[5,6]$.

In our study, we will work in the Banach space $B C\left(\mathbb{R}_{+}\right)$ consisting of all real, bounded, and continuous functions on $\mathbb{R}_{+}$. This space is furnished with the norm given by the formula

$$
\|x\|=\sup \left\{|x(t)|: t \in \mathbb{R}_{+}\right\} .
$$

In $\mathrm{BC}\left(\mathbb{R}_{+}\right)$, we will use the measure of noncompactness which appears in $[7,8]$. In order to present this measure of noncompactness, let us fix a nonempty, bounded subset $X$ of $\mathrm{BC}\left(\mathbb{R}_{+}\right)$and a number $L>0$. For $x \in X$ and $\varepsilon>0$, we denote by $\omega^{L}(x, \varepsilon)$ the modulus of continuity of the function $x$ on the interval $[0, L]$; that is,

$$
\omega^{L}(x, \varepsilon)=\sup \{|x(t)-x(s)|: t, s \in[0, L],|t-s| \leq \varepsilon\} .
$$

Now, we consider the quantities

$$
\begin{aligned}
\omega^{L}(X, \varepsilon) & =\sup \left\{\omega^{L}(x, \varepsilon): x \in X\right\}, \\
\omega_{0}^{L}(X) & =\lim _{\varepsilon \rightarrow 0} \omega^{L}(X, \varepsilon), \\
\omega_{0}(X) & =\lim _{L \rightarrow \infty} \omega_{0}^{L}(X) .
\end{aligned}
$$

Further, for a fixed number $t \in \mathbb{R}_{+}$, we denote $X(t)=\{x(t)$ : $x \in X\}$.

Finally, the measure of noncompactness $\mu$ which will be used in our study is defined as

$$
\mu(X)=\omega_{0}(X)+\limsup _{t \rightarrow \infty} \operatorname{diam} X(t),
$$

where $\operatorname{diam} X(t)=\sup \{|x(t)-y(t)|: x, y \in X\}$. In [4], the authors proved that the function $\mu$ is a measure of noncompactness in $\mathrm{BC}\left(\mathbb{R}_{+}\right)$.

In order to introduce the concept of asymptotic stability which will be used later, we assume that $\Omega$ is a nonempty subset of $\mathrm{BC}\left(\mathbb{R}_{+}\right)$and let $\mathscr{H}: \Omega \rightarrow \mathrm{BC}\left(\mathbb{R}_{+}\right)$be an operator. Also, consider the equation

$$
x(t)=(\mathscr{H} x)(t), \quad t \in \mathbb{R}_{+} .
$$

Definition 4. One will say that solutions of (8) are locally attractive if there exists a ball $B_{r}\left(x_{0}\right)$ in $\mathrm{BC}\left(\mathbb{R}_{+}\right)$such that, for arbitrary solutions $x=x(t)$ and $y=y(t)$ of (8) belonging to $B_{r}\left(x_{0}\right) \cap \Omega$, one has that

$$
\lim _{t \rightarrow \infty}(x(t)-y(t))=0 .
$$

In the case when the limit in (9) is uniform with respect to the set $B_{r}\left(x_{0}\right) \cap \Omega$, that is, when for each $\varepsilon>0$ there exists $T>0$ such that

$$
|x(t)-y(t)| \leq \varepsilon
$$

for all $x, y \in B_{r}\left(x_{0}\right) \cap \Omega$ being solutions of (8) and for any $t \geq T$, one will say that solutions of (8) are asymptotically stable.

We will finish this section with the following generalization of Banach contraction mapping principle due to Geraghty [9] and where the class $\mathscr{B}$ of functions $\beta$ : $[0, \infty) \rightarrow[0,1)$ is used satisfying

$$
\beta\left(t_{n}\right) \longrightarrow 1 \Longrightarrow t_{n} \longrightarrow 0
$$

By $\mathscr{B}_{0}$ we denote the class of functions $\beta:[0, \infty) \rightarrow[0,1)$.

Theorem 5. Let $(X, d)$ be a complete metric space and let $\mathscr{H}$ : $X \rightarrow X$ be an operator. Suppose that there exists $\beta \in \mathscr{B}$ such that for any $x, y \in X$

$$
d(\mathscr{H} x, \mathscr{H} y) \leq \beta(d(x, y)) \cdot d(x, y) .
$$

Then $\mathscr{H}$ has a unique fixed point in $X$.

\section{Main Result}

We start this section with the following result which is a version of Theorem 5 in the context of measure of noncompactness.

Proposition 6. Let $\Omega$ be a nonempty, bounded, closed, and convex subset of a Banach space $E$ and let $\mathscr{H}: \Omega \rightarrow \Omega$ be a continuous mapping such that

$$
\mu(\mathscr{H} X) \leq \beta(\mu(X)) \cdot \mu(X)
$$

for any nonempty and noncompact subset $X$ of $\Omega$, where $\beta \epsilon$ $\mathscr{B}_{0}$ and $\mu$ is an arbitrary measure of noncompactness in $E$. Then $\mathscr{H}$ has at least one fixed point. 
Proof. Let $X$ be a nonempty and noncompact subset of $\Omega$. Then $\mu(X)>0$. We can distinguish two cases.

Case $1(\beta(\mu(X))=0)$. In this case, from (13) we get $\mu(\mathscr{H} X)=$ 0 and therefore $0=\mu(\mathscr{H} X)<\mu(X)$.

Case $2(\beta(\mu(X))>0)$. In this case, since the function $\beta$ has as range $[0,1)$, from $(13)$ we have $\mu(\mathscr{H} X)<\mu(X)$.

Since $X$ is an arbitrary nonempty and noncompact subset of $\Omega$, the contractive condition appearing in Theorem 3 is satisfied. Finally, Theorem 3 says that $\mathscr{H}$ has a fixed point in $\Omega$. This completes the proof.

Now, we present the following result which belongs to the classical metric fixed point theory.

Corollary 7. Let $\Omega$ be a nonempty, bounded, closed, and convex subset of a Banach space $E$ and let $\mathscr{H}: \Omega \rightarrow \Omega$ be an operator satisfying

$$
\|\mathscr{H} x-\mathscr{H} y\| \leq \alpha(\|x-y\|)
$$

for any $x, y \in \Omega$, where $\alpha: \mathbb{R}_{+} \rightarrow \mathbb{R}_{+}$is a nondecreasing function with $\beta(t)=\alpha(t) / t \in \mathscr{B}_{0}$. Then $\mathscr{H}$ has a unique fixed point in $\Omega$.

Proof. Let $\mu: \mathfrak{M}_{E} \rightarrow \mathbb{R}_{+}$be the function defined by $\mu(X)=$ $\operatorname{diam} X$, where

$$
\operatorname{diam} X=\sup \{\|x-y\|: x, y \in X\} .
$$

It is easy to see that $\mu$ is a measure of noncompactness in $E$ [4].

Now, we take a nonempty subset $X$ of $\Omega$ with $\mu(X) \neq 0$. Using (14) and the fact that $\alpha$ is nondecreasing, we have

$$
\begin{aligned}
\mu(\mathscr{H} X) & =\operatorname{diam} \mathscr{H}(X)=\sup \{\|\mathscr{H} x-\mathscr{H} y\|: x, y \in X\} \\
& \leq \sup \{\alpha(\|x-y\|): x, y \in X\} \\
& \leq \alpha(\sup \{\|x-y\|: x, y \in X\}) \\
& =\alpha(\operatorname{diam} X)=\alpha(\mu(X))=\frac{\alpha(\mu(X))}{\mu(X)} \cdot \mu(X) \\
& =\beta(\mu(X)) \cdot \mu(X) .
\end{aligned}
$$

When $\mu(X)=\operatorname{diam} X=0$, we infer that $X$ is a singleton; thus $T X$ is also a singleton. Consequently, $\mu(\mathscr{H} X)=0$. Therefore, (16) is also satisfied when $\mu(X)=0$. Since $\beta \in \mathscr{B}_{0}$, Proposition 6 gives us the existence of at least one fixed point in $\Omega$.

In order to prove the uniqueness of the fixed point, we take into account Fix $\mathscr{H} \subset \operatorname{ker} \mu$, since $\mathscr{H}($ Fix $\mathscr{H})=$ Fix $\mathscr{H}$ and, consequently, $\mu($ Fix $\mathscr{H})=0$. Finally, since ker $\mu$ consists of singletons, Fix $\mathscr{H}$ is a singleton and this proves the uniqueness of the fixed point. The proof is complete.

An example of the function $\alpha$ appearing in Corollary 7 is $\alpha(t)=\arctan t$.

Now, we present the main result of the paper.
Theorem 8. Consider the following functional integral equation:

$$
x(t)=f(t, x(t))+\int_{0}^{t} g(t, s, x(s)) d s, \quad t \in \mathbb{R}_{+}
$$

under the following assumptions.

(a) The function $f(t, x)=f: \mathbb{R}_{+} \times \mathbb{R} \rightarrow \mathbb{R}$ is continuous and the function $t \rightarrow f(t, 0)$ is bounded.

(b) There exists a continuous and nondecreasing function $\varphi: \mathbb{R}_{+} \rightarrow \mathbb{R}_{+}$with $\varphi(0)=0$, satisfying

$\left(\mathrm{b}_{1}\right) \varphi((t+s) / 2) \geq(\varphi(t)+\varphi(s)) / 2$ for any $t, s \in \mathbb{R}_{+}$, $\left(\mathrm{b}_{2}\right) \beta(t)=\varphi(t) / t \in \mathscr{B}_{0}$,

such that

$|f(t, x)-f(t, y)| \leq \varphi(|x-y|) \quad$ for $x, y \in \mathbb{R}, t \in \mathbb{R}_{+}$.

(c) The function $g(t, s, x)=g: \mathbb{R}_{+}^{2} \times \mathbb{R} \rightarrow \mathbb{R}$ is continuous and there exist continuous functions $u, v$ : $\mathbb{R}_{+} \rightarrow \mathbb{R}_{+}$such that

$$
\begin{aligned}
& \left(\mathrm{c}_{1}\right) \lim _{t \rightarrow \infty} u(t) \int_{0}^{t} v(s) d s=0, \\
& \left(\mathrm{c}_{2}\right)|g(t, s, x)| \leq u(t) v(s) \text { for any } t, s \in \mathbb{R}_{+} \text {and } x \in \\
& \quad \mathbb{R} .
\end{aligned}
$$

(d) There exists a positive solution $r_{0}$ of the inequality $\varphi(r)+$ $q \leq r$, where

$$
q=\sup _{t \in \mathbb{R}_{+}}\left\{|f(t, 0)|+u(t) \int_{0}^{t} v(s) d s\right\} .
$$

Then (17) has at least one solution $x \in B C\left(\mathbb{R}_{+}\right)$. Moreover, solutions of (17) are asymptotically stable.

Proof. Let us consider the operator $\mathscr{H}$ defined on $\mathrm{BC}\left(\mathbb{R}_{+}\right)$as follows:

$$
(\mathscr{H} x)(t)=f(t, x(t))+\int_{0}^{t} g(t, s, x(s)) d s .
$$

For convenience, we divide the proof into several steps.

Step $1\left(\mathscr{H}\right.$ maps BC $\left(\mathbb{R}_{+}\right)$into itself). In fact, since $f$ and $g$ are continuous functions, for $x \in \mathrm{BC}\left(\mathbb{R}_{+}\right)$we infer that $\mathscr{H} x$ is continuous on $\mathbb{R}_{+}$. Now, we prove that for $x \in \mathrm{BC}\left(\mathbb{R}_{+}\right)$the function $\mathscr{H} x$ is bounded. In fact, for arbitrarily fixed $t \in \mathbb{R}_{+}$ we get

$$
\begin{aligned}
|(\mathscr{H} x)(t)| \leq & |f(t, x(t))-f(t, 0)| \\
& +|f(t, 0)|+\int_{0}^{t}|g(t, s, x(s))| d s \\
\leq & \varphi(|x(t)|)+|f(t, 0)|+u(t) \int_{0}^{t} v(s) d s \\
& \leq \varphi(\|x\|)+q .
\end{aligned}
$$


This proves that

$$
\sup _{t \in \mathbb{R}_{+}}|(\mathscr{H} x)(t)| \leq \varphi(\|x\|)+q<\infty .
$$

Therefore, $\mathscr{H}$ maps $\mathrm{BC}\left(\mathbb{R}_{+}\right)$into itself.

Step 2 ( $\mathscr{H}$ maps $B_{r_{0}}$ into itself). It follows from assumption (d) that $\mathscr{H}$ maps $B_{r_{0}}$ into itself.

Step 3 (an estimate of $\mathscr{H}$ with respect to the quantity $\omega_{0}$ ). For fixed $\varepsilon>0$ and $L>0$ let us take $t, s \in[0, L]$ with $|t-s| \leq \varepsilon$. Without loss of generality, we may assume that $s<t$. Then for $x \in X$ we obtain the following estimate:

$$
\begin{aligned}
& |(\mathscr{H} x)(t)-(\mathscr{H} x)(s)| \\
& \leq|f(t, x(t))-f(s, x(s))| \\
& +\left|\int_{0}^{t} g(t, \tau, x(\tau)) d \tau-\int_{0}^{s} g(s, \tau, x(\tau)) d \tau\right| \\
& \leq|f(t, x(t))-f(s, x(t))|+|f(s, x(t))-f(s, x(s))| \\
& +\left|\int_{0}^{t} g(t, \tau, x(\tau)) d \tau-\int_{0}^{t} g(s, \tau, x(\tau)) d \tau\right| \\
& +\left|\int_{0}^{t} g(s, \tau, x(\tau)) d \tau-\int_{0}^{s} g(s, \tau, x(\tau)) d \tau\right| \\
& \leq \omega_{1}^{L}(f, \varepsilon)+\varphi(|x(t)-x(s)|) \\
& +\int_{0}^{t}|g(t, \tau, x(\tau))-g(s, \tau, x(\tau))| d \tau \\
& +\int_{s}^{t}|g(s, \tau, x(\tau))| d \tau \\
& \leq \omega_{1}^{L}(f, \varepsilon)+\varphi\left(\omega^{L}(x, \varepsilon)\right) \\
& +\int_{0}^{L} \omega_{1}^{L}(g, \varepsilon) d \tau+u(s) \int_{s}^{t} v(\tau) d \tau \\
& \leq \omega_{1}^{L}(f, \varepsilon)+\varphi\left(\omega^{L}(x, \varepsilon)\right) \\
& +L \omega_{1}^{L}(g, \varepsilon)+\varepsilon \sup _{s, t \in[0, L]}\{u(s) v(t)\},
\end{aligned}
$$

where we denoted

$$
\begin{array}{r}
\omega_{1}^{L}(f, \varepsilon)=\sup \{|f(t, x)-f(s, x)|: t, s \in[0, L], \\
\left.x \in\left[-r_{0}, r_{0}\right],|t-s| \leq \varepsilon\right\}, \\
\omega_{1}^{L}(g, \varepsilon)=\sup \{|g(t, \tau, x)-g(s, \tau, x)|: t, \tau, s \in[0, L], \\
\left.x \in\left[-r_{0}, r_{0}\right],|t-s| \leq \varepsilon\right\} .
\end{array}
$$

From the uniform continuity of the functions $f$ and $g$ on the sets $[0, L] \times\left[-r_{0}, r_{0}\right]$ and $[0, L] \times[0, L] \times\left[-r_{0}, r_{0}\right]$, respectively, it follows that $\omega_{1}^{L}(f, \varepsilon) \rightarrow 0$ and $\omega_{1}^{L}(g, \varepsilon) \rightarrow 0$ when $\varepsilon \rightarrow 0$. Notice that, since $u$ and $v$ are continuous on $[0, L]$, we have that $\sup _{s, t \in[0, L]}\{u(s) v(t)\}<\infty$. Therefore, we derive the following estimate:

$$
\begin{aligned}
\omega^{L}(\mathscr{H} x, \varepsilon) \leq & \omega_{1}^{L}(f, \varepsilon)+\varphi\left(\omega^{L}(x, \varepsilon)\right) \\
& +L \omega_{1}^{L}(g, \varepsilon)+\varepsilon \sup _{s, t \in[0, L]}\{u(s) v(t)\} .
\end{aligned}
$$

Since $\varphi$ is nondecreasing, we obtain

$$
\begin{aligned}
\omega^{L}(\mathscr{H} X, \varepsilon)= & \sup _{x \in X}\left\{\omega^{L}(\mathscr{H} x, \varepsilon)\right\} \\
\leq & \omega_{1}^{L}(f, \varepsilon)+\varphi\left(\sup _{x \in X}\left\{\omega^{L}(x, \varepsilon)\right\}\right) \\
& +L \omega_{1}^{L}(g, \varepsilon)+\varepsilon \sup _{s, t \in[0, L]}\{u(s) v(t)\} \\
= & \omega_{1}^{L}(f, \varepsilon)+\varphi\left(\omega^{L}(X, \varepsilon)\right) \\
& +L \omega_{1}^{L}(g, \varepsilon)+\varepsilon \sup _{s, t \in[0, L]}\{u(s) v(t)\} .
\end{aligned}
$$

Hence

$$
\begin{aligned}
\omega_{0}^{L}(\mathscr{H} X) & =\lim _{\varepsilon \rightarrow 0} \omega^{L}(\mathscr{H} X, \varepsilon) \leq \lim _{\varepsilon \rightarrow 0} \varphi\left(\omega^{L}(X, \varepsilon)\right) \\
& =\varphi\left(\lim _{\varepsilon \rightarrow 0} \omega^{L}(X, \varepsilon)\right)=\varphi\left(\omega_{0}^{L}(X)\right) .
\end{aligned}
$$

Finally, we get

$$
\begin{aligned}
\omega_{0}(\mathscr{H} X) & =\lim _{L \rightarrow \infty} \omega_{0}^{L}(\mathscr{H} X) \leq \lim _{L \rightarrow \infty} \varphi\left(\omega_{0}^{L}(X)\right) \\
& =\varphi\left(\lim _{L \rightarrow \infty} \omega_{0}^{L}(X)\right)=\varphi\left(\omega_{0}(X)\right) .
\end{aligned}
$$

Step 4 (an estimate of $\mathscr{H}$ with respect to the diameter). For $x, y \in X$ and $t \in \mathbb{R}_{+}$, we have

$$
\begin{aligned}
& |(\mathscr{H} x)(t)-(\mathscr{H} y)(t)| \\
& \leq|f(t, x(t))-f(t, y(t))| \\
& \quad+\int_{0}^{t}|g(t, s, x(s))| d s+\int_{0}^{t}|g(t, s, y(s))| d s \\
& \leq \varphi(|x(t)-y(t)|)+2 u(t) \int_{0}^{t} v(s) d s .
\end{aligned}
$$

Since $\varphi$ is nondecreasing, from the last inequality it follows that

$$
\operatorname{diam}(\mathscr{H} X)(t) \leq \varphi(\operatorname{diam} X(t))+2 u(t) \int_{0}^{t} v(s) d s .
$$

Consequently, from assumption $\left(c_{1}\right)$ and the continuity of $\varphi$, we get

$$
\limsup _{t \rightarrow \infty} \operatorname{diam}(\mathscr{H} X)(t) \leq \varphi\left(\limsup _{t \rightarrow \infty} \operatorname{diam} X(t)\right) .
$$


Step 5 ( $\mathscr{H}$ satisfies the contractive condition of Proposition 6). In fact, from assumption $\left(b_{1}\right),(28),(31)$, and the definition of the measure of noncompactness $\mu$, we infer

$$
\begin{aligned}
\mu(\mathscr{H} X) & =\omega_{0}(\mathscr{H} X)+\limsup _{t \rightarrow \infty} \operatorname{diam}(\mathscr{H} X)(t) \\
& \leq \varphi\left(\omega_{0}(X)\right)+\varphi\left(\limsup _{t \rightarrow \infty} \operatorname{diam} X(t)\right) \\
& =2 \frac{\varphi\left(\omega_{0}(X)\right)+\varphi\left(\limsup _{t \rightarrow \infty} \operatorname{diam} X(t)\right)}{2} \\
& \leq 2 \varphi\left(\frac{\omega_{0}(X)+\lim \sup _{t \rightarrow \infty} \operatorname{diam} X(t)}{2}\right) \\
& =2 \varphi\left(\frac{\mu(X)}{2}\right) .
\end{aligned}
$$

Now, considering in $\mathrm{BC}\left(\mathbb{R}_{+}\right)$the measure of noncompactness $\mu_{1}$ defined by $\mu_{1}(X)=(1 / 2) \mu(X)$, the last estimate can be written in the form

$$
\mu_{1}(\mathscr{H} X) \leq \varphi\left(\mu_{1}(X)\right) .
$$

Therefore, if $\mu_{1}(X) \neq 0$, then

$$
\mu_{1}(\mathscr{H} X) \leq \frac{\varphi\left(\mu_{1}(X)\right)}{\mu_{1}(X)} \cdot \mu_{1}(X)
$$

or equivalently

$$
\mu_{1}(\mathscr{H} X) \leq \beta\left(\mu_{1}(X)\right) \cdot \mu_{1}(X),
$$

where $\beta(t)=\varphi(t) / t$

In the case $\mu_{1}(X)=0$ we have that $X$ is a relatively compact subset of $\mathrm{BC}\left(\mathbb{R}_{+}\right)$and, since $\mathscr{H}$ is continuous, $\mathscr{H} X$ is also relatively compact and thus $\mu_{1}(\mathscr{H} X)=0$. This proves that (35) is also satisfied when $\mu_{1}(X)=0$. Summarizing, for any nonempty subset $X$ of $B_{r_{0}}$, we have

$$
\mu_{1}(\mathscr{H} X) \leq \beta\left(\mu_{1}(X)\right) \cdot \mu_{1}(X),
$$

where $\beta \in \mathscr{B}_{0}$ (assumption $\left(\mathrm{b}_{2}\right)$ ) and $\mu_{1}$ is a measure of noncompactness in $\mathrm{BC}\left(\mathbb{R}_{+}\right)$.

In the sequel, let us consider the sequence of sets $\left(B_{r_{0}}^{n}\right)$, where $B_{r_{0}}^{1}=\operatorname{Conv} \mathscr{H}\left(B_{r_{0}}\right), B_{r_{0}}^{2}=\operatorname{Conv} \mathscr{H}\left(B_{r_{0}}^{1}\right)$, and so on. Notice that the sequence is decreasing; that is, $B_{r_{0}}^{n+1} \subset B_{r_{0}}^{n}$ for $n=1,2,3, \ldots$. Moreover, $B_{r_{0}}^{1} \subset B_{r_{0}}$ and the sets in this sequence are closed, convex, and nonempty.
On the other hand, in view of (32), we get

$$
\begin{aligned}
\mu_{1}\left(B_{r_{0}}^{1}\right) & =\mu_{1}\left(\operatorname{Conv} \mathscr{H}\left(B_{r_{0}}\right)\right) \\
& =\mu_{1}\left(\mathscr{H}\left(B_{r_{0}}\right)\right) \\
& \leq \varphi\left(\mu_{1}\left(B_{r_{0}}\right)\right), \\
\mu_{1}\left(B_{r_{0}}^{2}\right) & =\mu_{1}\left(\operatorname{Conv} \mathscr{H}\left(B_{r_{0}}^{1}\right)\right) \\
& =\mu_{1}\left(\mathscr{H}\left(B_{r_{0}}^{1}\right)\right) \\
& \leq \varphi\left(\mu_{1}\left(B_{r_{0}}^{1}\right)\right) \\
& \leq \varphi\left(\varphi\left(\mu_{1}\left(B_{r_{0}}\right)\right)\right) \\
& =\varphi^{2}\left(\mu_{1}\left(B_{r_{0}}\right)\right)
\end{aligned}
$$

and, by using induction,

$$
\mu_{1}\left(B_{r_{0}}^{n}\right) \leq \varphi^{n}\left(\mu_{1}\left(B_{r_{0}}\right)\right)
$$

where we have used the nondecreasing character of $\varphi$ and where $\varphi^{(n)}$ denotes the $n$th iteration. Taking into account $\left(b_{2}\right)$, since $\varphi(t) / t \in \mathscr{B}_{0}$, we have $\varphi(t)<t$ for $t>0$ and as $\varphi$ is continuous, it follows that $\varphi^{(n)}(t) \rightarrow 0$ for $t>0$ [10].

Therefore, we deduce that

$$
\lim _{n \rightarrow \infty} \mu_{1}\left(B_{r_{0}}^{n}\right)=\lim _{n \rightarrow \infty} \varphi^{n}\left(\mu_{1}\left(B_{r_{0}}\right)\right)=0 .
$$

Now, taking into account Definition 1, we deduce that the set $Y=\bigcap_{n=1}^{\infty} B_{r_{0}}^{n}$ is nonempty, bounded, closed, and convex. Moreover, since $\mu_{1}(Y) \leq \mu_{1}\left(B_{r_{0}}^{n}\right)$ for any $n \in \mathbb{N}, Y$ is a member of the kernel ker $\mu_{1}$ of the measure of noncompactness $\mu_{1}$. Let us also observe that the operator $\mathscr{H}$ transforms the set $Y$ into itself.

Next, we will prove that $\mathscr{H}$ is continuous on the set $Y$. To do this let us fix a number $\varepsilon>0$ and we take a sequence $\left(x_{n}\right) \subset Y$ and $x \in Y$ such that $x_{n} \rightarrow x$. We have to prove that $\mathscr{H} x_{n} \rightarrow \mathscr{H} x$.

In fact, since $Y \in \operatorname{ker} \mu_{1}$, we have $\mu_{1}(Y)=0$ and, particularly, $\lim \sup _{t \rightarrow \infty} \operatorname{diam} Y(t)=0$. Then, for $\varepsilon>0$ we can find $T>0$ such that $|x(t)-y(t)|<\varepsilon$ for any $x, y \in Y$ and $t \geq T$. Particularly, since $\mathscr{H}: Y \rightarrow Y$ we have $\mathscr{H} x_{n}, \mathscr{H} x \in Y$ for any $n \in \mathbb{N}$, and, thus, for $t \geq T$,

$$
\left|\left(\mathscr{H} x_{n}\right)(t)-(\mathscr{H} x)(t)\right|<\varepsilon, \quad \text { for any } n \in \mathbb{N} \text {. }
$$

On the other hand, since $g:[0, T] \times[0, T] \times\left[-r_{0}, r_{0}\right] \rightarrow \mathbb{R}$ is continuous on a compact set, it is uniformly continuous. This means that for $\varepsilon>0$ we can find $\delta>0$ such that if $\max \left\{\left|t-t^{\prime}\right|,\left|s-s^{\prime}\right|,|u-v|\right\}<\delta$ for $t, t^{\prime}, s, s^{\prime} \in[0, T]$ and $u, v \in\left[-r_{0}, r_{0}\right]$, we have $\left|g(t, s, u)-g\left(t^{\prime}, s^{\prime}, v\right)\right|<\varepsilon / 2 T$.

Taking into account that $x_{n} \rightarrow x$, we can find $n_{0} \in \mathbb{N}$ such that, for $n \geq n_{0},\left\|x_{n}-x\right\|<\min \{\varepsilon / 2, \delta\}$. 
For $n \geq n_{0}$ and $t \in[0, T]$, we have

$$
\begin{aligned}
\left|\left(\mathscr{H} x_{n}\right)(t)-(\mathscr{H} x)(t)\right| & \\
\leq & \left|f\left(t, x_{n}(t)\right)-f(t, x(t))\right| \\
& \quad+\int_{0}^{t}\left|g\left(t, s, x_{n}(s)\right)-g(t, s, x(s))\right| d s \\
\leq & \varphi\left(\left|x_{n}(t)-x(t)\right|\right) \\
& \quad+\int_{0}^{T}\left|g\left(t, s, x_{n}(s)\right)-g(t, s, x(s))\right| d s \\
\leq & \varphi\left(\left\|x_{n}-x\right\|\right)+\int_{0}^{T} \frac{\varepsilon}{2 T} d s \\
\leq & \varphi\left(\frac{\varepsilon}{2}\right)+\frac{\varepsilon}{2} \\
< & \frac{\varepsilon}{2}+\frac{\varepsilon}{2} \\
= & \varepsilon
\end{aligned}
$$

where we have used the fact that $\varphi(t)<t$ for $t>0$ and the nondecreasing character of $\varphi$.

From (40) and (41), $\left\|\mathscr{H} x_{n}-\mathscr{H} x\right\|<\varepsilon$ for $n \geq n_{0}$. This proves our claim.

Finally, taking into account that as $Y \in \operatorname{ker} \mu_{1}$ and, consequently, $Y$ is relatively compact, $\mathscr{H}: Y \rightarrow Y$ is a continuous operator, applying the classical Schauder fixed point theorem, we infer that the operator $\mathscr{H}$ has at least one fixed point in $Y$.

In order to prove that solutions of (17) are asymptotically stable, we notice that any solution $x(t)$ of (17) in $B_{r_{0}}$ is a fixed point of $\mathscr{H}$. Now, taking into account that $\mathscr{H}$ transforms $B_{r_{0}}$ into itself, we have

$$
\begin{aligned}
\mu_{1}\left(\mathscr{H}\left(B_{r_{0}} \cap \operatorname{Fix} \mathscr{H}\right)\right) & =\mu_{1}\left(B_{r_{0}} \cap \mathscr{H}(\operatorname{Fix} \mathscr{H})\right) \\
& =\mu_{1}\left(B_{r_{0}} \cap \operatorname{Fix} \mathscr{H}\right) .
\end{aligned}
$$

Since $\mu_{1}(\mathscr{H} X) \leq \beta\left(\mu_{1}(X)\right) \cdot \mu_{1}(X)$ for any nonempty subset $X$ of $B_{r_{0}}$, we have

$$
\begin{aligned}
\mu_{1}\left(\mathscr{H}\left(B_{r_{0}} \cap \operatorname{Fix} \mathscr{H}\right)\right)= & \mu_{1}\left(B_{r_{0}} \cap \operatorname{Fix} \mathscr{H}\right) \\
\leq & \beta\left(\mu_{1}\left(B_{r_{0}} \cap \operatorname{Fix} \mathscr{H}\right)\right) \\
& \cdot \mu_{1}\left(B_{r_{0}} \cap \operatorname{Fix} \mathscr{H}\right) .
\end{aligned}
$$

Further, we distinguish two cases.

Case $1\left(\beta\left(\mu_{1}\left(B_{r_{0}} \cap\right.\right.\right.$ Fix $\left.\left.\left.\mathscr{H}\right)\right)=0\right)$. In this case, by $(43), \mu_{1}\left(B_{r_{0}} \cap\right.$ Fix $\mathscr{H})=0$.
Case $2\left(\beta\left(\mu_{1}\left(B_{r_{0}} \cap\right.\right.\right.$ Fix $\left.\left.\left.\mathscr{H}\right)\right)>0\right)$. In this case, by $(43)$ and taking into account that the range of the function $\beta$ is $[0,1)$, we infer

$$
\begin{aligned}
\mu_{1} & \left(\mathscr{H}\left(B_{r_{0}} \cap \operatorname{Fix} \mathscr{H}\right)\right) \\
& =\mu_{1}\left(B_{r_{0}} \cap \operatorname{Fix} \mathscr{H}\right) \\
& \leq \beta\left(\mu_{1}\left(B_{r_{0}} \cap \operatorname{Fix} \mathscr{H}\right)\right) \cdot \mu_{1}\left(B_{r_{0}} \cap \operatorname{Fix} \mathscr{H}\right) \\
& <\mu_{1}\left(B_{r_{0}} \cap \operatorname{Fix} \mathscr{H}\right)
\end{aligned}
$$

which is a contradiction. Therefore $\mu_{1}\left(B_{r_{0}} \cap\right.$ Fix $\left.\mathscr{H}\right)=0$.

Since $\mu_{1}(X)=(1 / 2) \mu(X)$ for any nonempty subset $X$, we deduce that $\mu\left(B_{r_{0}} \cap\right.$ Fix $\left.\mathscr{H}\right)=0$. Taking into account the definition of the measure of noncompactness $\mu$ (see Section 2), we have

$$
\limsup _{t \rightarrow \infty} \operatorname{diam}\left[\left(B_{r_{0}} \cap \operatorname{Fix} \mathscr{H}\right)(t)\right]=0 .
$$

But this means that for any $\varepsilon>0$ we can find $T>0$ such that

$$
\operatorname{diam}\left[\left(B_{r_{0}} \cap \operatorname{Fix} \mathscr{H}\right)(t)\right] \leq \varepsilon \quad \text { for any } t \geq T \text {. }
$$

As all solutions of (17) in $B_{r_{0}}$ are in $B_{r_{0}} \cap$ Fix $\mathscr{H}$, by (46) we have that for $\varepsilon>0$ there exists $T>0$ such that

$$
|x(t)-y(t)| \leq \varepsilon \quad \text { for any } t \geq T,
$$

where $x, y \in B_{r_{0}}$ and they are solutions of (17). This means that solutions of (17) are asymptotically stable. The proof is complete.

\section{Example}

In order to present an example which illustrates our results, we need to prove some properties about the inverse tangent function.

Lemma 9. The function $\varphi: \mathbb{R}_{+} \rightarrow[0, \pi / 2)$ defined as $\varphi(t)=$ $\arctan t$ has the following properties.

(a) $\varphi$ is continuous, nondecreasing and satisfies $\varphi(0)=0$.

(b) For $t, s \in \mathbb{R}_{+}:(\varphi(t)+\varphi(s)) / 2 \leq \varphi((t+s) / 2)$.

Proof. (a) It is clear that $\varphi$ is continuous, nondecreasing (since $\left.\varphi^{\prime}(t)=1 /\left(1+t^{2}\right)>0\right)$ and $\varphi(0)=0$.

(b) Since $\varphi^{\prime \prime}(t)=-2 t /\left(1+t^{2}\right)^{2} \leq 0$ for any $t \in \mathbb{R}_{+}$, we infer that $\varphi$ is concave and, therefore,

$$
\frac{\varphi(t)+\varphi(s)}{2} \leq \varphi\left(\frac{t+s}{2}\right)
$$

for any $t, s \in \mathbb{R}_{+}$.

Definition 10. A function $f: \mathbb{R}_{+} \rightarrow \mathbb{R}_{+}$is said to be subadditive if

$$
f(x+y) \leq f(x)+f(y), \quad \text { for any } x, y \in \mathbb{R}_{+} .
$$


Lemma 11. Suppose that $f: \mathbb{R}_{+} \rightarrow \mathbb{R}_{+}$is subadditive. Then, for $y \leq x$, one has that

$$
f(x)-f(y) \leq f(x-y) .
$$

Proof. In fact, since

$$
f(x)=f(x-y+y) \leq f(x-y)+f(y)
$$

the desired result follows.

Remark 12. From Lemma 11, we infer that if $f: \mathbb{R}_{+} \rightarrow \mathbb{R}_{+}$is subadditive, then

$$
|f(x)-f(y)| \leq f(|x-y|), \quad \text { for any } x, y \in \mathbb{R}_{+} .
$$

Lemma 13. Let $f: \mathbb{R}_{+} \rightarrow \mathbb{R}_{+}$be a concave function with $f(0)=0$. Then $f$ is subadditive.

Proof. Since $f$ is concave and $f(0)=0$, we have for $x, y \in \mathbb{R}_{+}$

$$
\begin{aligned}
f(x) & =f\left(\frac{x}{x+y}(x+y)+\frac{y}{x+y} \cdot 0\right) \\
& \geq \frac{x}{x+y} f(x+y)+\frac{y}{x+y} f(0) \\
& =\frac{x}{x+y} f(x+y), \\
f(y) & =f\left(\frac{x}{x+y} \cdot 0+\frac{y}{x+y}(x+y)\right) \\
& \geq \frac{x}{x+y} f(0)+\frac{y}{x+y} f(x+y) \\
& =\frac{y}{x+y} f(x+y) .
\end{aligned}
$$

Adding these inequalities side by side, we obtain

$$
\begin{aligned}
f(x)+f(y) & \geq \frac{x}{x+y} f(x+y)+\frac{y}{x+y} f(x+y) \\
& =f(x+y) .
\end{aligned}
$$

Therefore $f$ is subadditive.

Remark 14. Since the function $f: \mathbb{R}_{+} \rightarrow[0, \pi / 2)$ defined by $\varphi(t)=\arctan t$ is concave and $\varphi(0)=0$, by Lemma $13, \varphi$ is subadditive. Taking into account Remark 12, we get

$$
|\varphi(t)-\varphi(s)| \leq \varphi(|t-s|) \quad \text { for any } t, s \in \mathbb{R}_{+}
$$

or

$$
|\arctan t-\arctan s| \leq \arctan (|t-s|) \quad \text { for any } t, s \in \mathbb{R}_{+} .
$$

Lemma 15. The function $\beta: \mathbb{R}_{+} \rightarrow[0,1)$ defined by $\beta(t)=$ $\varphi(t) / t$ for $t>0$, where $\varphi(t)=\arctan t$, belongs to the class $\mathscr{B}_{0}$.

Proof. From mathematical analysis, we know that $\varphi(t)=$ $\arctan t<t$, for $t>0$. Therefore, the function $\varphi$ maps $\mathbb{R}_{+}$ into $[0,1)$. This completes the proof.
Now, we are ready to present an example illustrating our results.

Example 16. Consider the following functional integral equation:

$$
\begin{aligned}
x(t)= & \frac{1}{20} \frac{t}{1+t}+\arctan x(t) \\
& +\int_{0}^{t} \frac{t s \cos \left(x^{2}(t)\right)}{30 \sqrt{t^{8}+s^{2}+t^{8} s^{2}+2}} d s, \quad t \in \mathbb{R}_{+} .
\end{aligned}
$$

Notice that (57) is a particular case of (17), provided we put

$$
\begin{gathered}
f(t, x)=\frac{1}{20} \frac{t}{1+t}+\arctan x, \\
g(t, s, x)=\frac{t s \cos \left(x^{2}\right)}{30 \sqrt{t^{8}+s^{2}+t^{8} s^{2}+2}} .
\end{gathered}
$$

It is clear that $f: \mathbb{R}_{+} \times \mathbb{R} \rightarrow \mathbb{R}$ is continuous and, moreover,

$$
|f(t, 0)|=\left|\frac{1}{20} \frac{t}{1+t}\right| \leq \frac{1}{20}, \quad \text { for any } t \in \mathbb{R}_{+} \text {. }
$$

Thus, assumption (a) of Theorem 8 is satisfied.

On the other hand, taking into account Remark 14, for any $x, y \in \mathbb{R}$ and $t \in \mathbb{R}_{+}$, we obtain

$$
\begin{aligned}
|f(t, x)-f(t, y)| & =|\arctan x-\arctan y| \\
& \leq \arctan (|x-y|) .
\end{aligned}
$$

Thus, by Lemmas 9 and 15, the function $\varphi(t)=\arctan t$ satisfies assumption (b) of Theorem 8.

Further, notice that $g: \mathbb{R}_{+} \times \mathbb{R}_{+} \times \mathbb{R} \rightarrow \mathbb{R}$ is continuous and, for any $t, s \in \mathbb{R}_{+}$and for $x \in \mathbb{R}$, one has

$$
\begin{aligned}
|g(t, s, x)| & =\left|\frac{t s \cos \left(x^{2}\right)}{30 \sqrt{t^{8}+s^{2}+t^{8} s^{2}+2}}\right| \\
& \leq \frac{t}{30 \sqrt{t^{8}+1}} \cdot \frac{s}{\sqrt{s^{2}+1}} .
\end{aligned}
$$

Putting $u(t)=t / 30 \sqrt{t^{8}+1}$ and $v(s)=s / \sqrt{s^{2}+1}$, it is clear that $u(t)$ and $v(s)$ are continuous functions on $\mathbb{R}_{+}$. Moreover,

$$
\int_{0}^{t} v(s) d s=\int_{0}^{t} \frac{s}{\sqrt{s^{2}+1}}=\sqrt{t^{2}+1}-1 .
$$

Since

$$
\lim _{t \rightarrow \infty} u(t) \int_{0}^{t} v(s) d s=\lim _{t \rightarrow \infty} \frac{t}{30 \sqrt{t^{8}+1}}\left(\sqrt{t^{2}+1}-1\right)=0,
$$

assumption (c) of Theorem 8 is satisfied. 
Now, we estimate the constant $q$ appearing in assumption (d) of Theorem 8 . Indeed, we have

$$
\begin{aligned}
q & =\sup _{t \in \mathbb{R}_{+}}\left\{|f(t, 0)|+u(t) \int_{0}^{t} v(s) d s\right\} \\
& =\sup _{t \in \mathbb{R}_{+}}\left\{\frac{1}{20} \frac{t}{1+t}+\frac{t}{30 \sqrt{t^{8}+1}}\left(\sqrt{t^{2}+1}-1\right)\right\} \\
& \leq \sup _{t \in \mathbb{R}_{+}}\left\{\frac{1}{20}+\frac{t}{30 \sqrt{t^{8}+1}} \sqrt{t^{2}+1}\right\} \\
& =\sup _{t \in \mathbb{R}_{+}}\left\{\frac{1}{20}+\frac{\sqrt{t^{4}+t^{2}}}{30 \sqrt{t^{8}+1}}\right\} .
\end{aligned}
$$

Notice that if $t<1$, then

$$
\frac{\sqrt{t^{4}+t^{2}}}{\sqrt{t^{8}+1}} \leq \frac{\sqrt{2}}{\sqrt{t^{8}+1}} \leq \sqrt{2}
$$

and when $t \geq 1$, we have

$$
\frac{\sqrt{t^{4}+t^{2}}}{\sqrt{t^{8}+1}} \leq \frac{\sqrt{2 t^{8}}}{\sqrt{t^{8}+1}} \leq \sqrt{2} .
$$

Thus, from the last inequality, we obtain

$$
\begin{aligned}
q & \leq \sup _{t \in \mathbb{R}_{+}}\left\{\frac{1}{20}+\frac{\sqrt{t^{4}+t^{2}}}{30 \sqrt{t^{8}+1}}\right\} \\
& \leq \frac{1}{20}+\frac{1}{30} \sqrt{2}<0.0972 .
\end{aligned}
$$

Now, we consider the inequality

$$
\varphi(r)+0.0972=\arctan r+0.0972<r .
$$

An application of Bolzano's theorem gives that this inequality is satisfied by a number $r_{0} \in(0,1)$. Therefore, assumption (d) of Theorem 8 is satisfied.

Finally, by Theorem 8 , we conclude that (57) has at least one solution $x$ in $\mathrm{BC}\left(\mathbb{R}_{+}\right)$satisfying $\|x\| \leq r_{0}$.

\section{Final Remarks}

In [10] the authors proved the following result.

Theorem 17 (Theorem 2.2 of [10]). Let $\Omega$ be a nonempty, bounded, closed, and convex subset of a Banach space $E$ and let $T: \Omega \rightarrow \Omega$ be a continuous operator satisfying

$$
\mu(T X) \leq \varphi(\mu(X))
$$

for any nonempty subset $X$ of $\Omega$, where $\mu$ is an arbitrary measure of noncompactness and $\varphi: \mathbb{R}_{+} \rightarrow \mathbb{R}_{+}$is a nondecreasing function such that $\lim _{n \rightarrow \infty} \varphi^{n}(t)=0$ for each $t \in \mathbb{R}_{+}$, where $\varphi^{n}$ denotes the $n$-iteration of $\varphi$. Then $T$ has at least one fixed point in $\Omega$.
Notice that we can rewrite condition (69) in the form

$$
\mu(T X) \leq \frac{\varphi(\mu(X))}{\mu(X)} \cdot \mu(X),
$$

for any nonempty subset $X$ of $\Omega$ with $\mu(X)>0$. When $\mu(X)=$ 0 , this means that $X$ is a relatively compact subset of $E$ and, since $T$ is continuous, $T X$ is also relatively compact subset of $E$ and, therefore, $\mu(T X)=0$. Consequently, condition (70) is satisfied for any nonempty subset $X$ of $\Omega$. This tells us that Theorem 2.2 of [10] can be reformulated in the following way.

Theorem 18. Let $\Omega$ be a nonempty, bounded, closed, and convex subset of a Banach space $E$ and let $T: \Omega \rightarrow \Omega$ be a continuous operator satisfying

$$
\mu(T X) \leq \gamma(\mu(X)) \cdot \mu(X),
$$

for any nonempty subset $X$ of $\Omega$, where $\mu$ is an arbitrary measure of noncompactness and $\gamma$ belongs to the class $\mathscr{A}$ of functions $\phi: \mathbb{R}_{+} \rightarrow \mathbb{R}_{+}$with $\phi(t)=\varphi(t) / t$, where $\varphi: \mathbb{R}_{+} \rightarrow$ $\mathbb{R}_{+}$is a nondecreasing function such that $\lim _{n \rightarrow \infty} \varphi^{n}(t)=0$ for each $t \in \mathbb{R}_{+}$. Then $T$ has at least one fixed point in $\Omega$.

Now, we compare the classes of functions $\mathscr{B}_{0}$ and $\mathscr{A}$ appearing in Proposition 6 and Theorem 18, respectively. To do this, we need the following lemma which appears in [10] under weaker assumptions. For the paper to be selfcontained, we present a proof.

Lemma 19. Let $\varphi: \mathbb{R}_{+} \rightarrow \mathbb{R}_{+}$be a continuous and nondecreasing function. Then the following conditions are equivalent:
(a) $\lim _{n \rightarrow \infty} \varphi^{n}(t)=0$ for any $t>0$,
(b) $\varphi(t)<t$ for any $t>0$.

Proof. (a) $\Rightarrow$ (b) Suppose that the conclusion is not true. This means that we can find $t_{0}>0$ such that $\varphi\left(t_{0}\right) \geq t_{0}$. Since $\varphi$ is nondecreasing, we obtain $\varphi^{n}\left(t_{0}\right) \geq t_{0}>0$ for any $n=$ $1,2, \ldots$ and the sequence $\left\{\varphi^{n}\left(t_{0}\right)\right\}$ is nondecreasing. Therefore $\lim _{n \rightarrow \infty} \varphi^{n}\left(t_{0}\right) \geq t_{0}>0$ and this contradicts (a).

(b) $\Rightarrow$ (a) Let $t$ be an arbitrary number but fixed with $t>$ 0 . Since $\varphi(t)<t$ and $\varphi$ is nondecreasing we infer that the sequence of nonnegative real numbers $\left(\varphi^{n}(t)\right)$ is decreasing. Thus, $\lim _{n \rightarrow \infty} \varphi^{n}(t)=r$ for certain $r \geq 0$. Suppose that $r>0$. Then, by (b), $\varphi(r)<r$. On the other hand, since $r \leq \varphi^{n}(t)$ for any $n=1,2, \ldots$, the continuity of $\varphi$ gives us

$$
\begin{aligned}
r & \leq \lim _{n \rightarrow \infty} \varphi^{n}(t)=\lim _{n \rightarrow \infty} \varphi\left(\varphi^{n-1}(t)\right) \\
& =\varphi\left(\lim _{n \rightarrow \infty} \varphi^{n-1}(t)\right)=\varphi(r)<r,
\end{aligned}
$$

which leads to a contradiction. Therefore, $r=0$ and this completes the proof. In virtue of Lemma 19, it is obvious that $\mathscr{A} \subset \mathscr{B}_{0}$. 
Now, we will prove that $\mathscr{B}_{0} \not \subset \mathscr{A}$. To this end consider the function $\gamma: \mathbb{R}_{+} \rightarrow \mathbb{R}_{+}$given by

$$
\gamma(t)= \begin{cases}-\frac{1}{2} t+\frac{1}{2}, & \text { if } 0 \leq t \leq 1 \\ 0, & \text { if } t>1\end{cases}
$$

It is clear that $\gamma$ maps $\mathbb{R}_{+}$into $[0,1 / 2]$ and therefore $\gamma \in \mathscr{B}$.

If $\gamma \in \mathscr{A}$, then $\gamma(t)=\varphi(t) / t$, where $\varphi: \mathbb{R}_{+} \rightarrow \mathbb{R}_{+}$is a nondecreasing and $\lim _{n \rightarrow \infty} \varphi^{n}(t)=0$ for $t \in \mathbb{R}_{+}$. In view of the equality

$$
\varphi(t)=t \gamma(t)= \begin{cases}-\frac{1}{2} t^{2}+\frac{1}{2} t, & \text { if } 0 \leq t \leq 1 \\ 0, & \text { if } t>1\end{cases}
$$

it is obvious that $\varphi$ is not nondecreasing and, consequently, $\gamma \notin \mathscr{A}$. This proves that $\mathscr{B}_{0} \not \subset \mathscr{A}$.

In [7], the authors investigated the following functional integral equation:

$$
\begin{aligned}
x(t)= & f(t, x(\alpha(t))) \\
& +\int_{0}^{\beta(t)} g(t, s, x(\gamma(s))) d s, \quad t \in \mathbb{R}_{+},
\end{aligned}
$$

under the following assumptions.

(i) The functions $\alpha, \beta, \gamma: \mathbb{R}_{+} \rightarrow \mathbb{R}_{+}$are continuous and $\alpha(t) \rightarrow \infty$ as $t \rightarrow \infty$.

(ii) The function $f: \mathbb{R}_{+} \times \mathbb{R} \rightarrow \mathbb{R}$ is continuous and there exist positive constants $L, M$ such that

$$
|f(t, x)-f(t, y)| \leq \frac{M|x-y|}{L+|x-y|}
$$

for $t \in \mathbb{R}_{+}$and for $x, y \in \mathbb{R}$. Moreover, we assume that $M<L$.

(iii) The function $t \rightarrow f(t, 0)$ is bounded on $\mathbb{R}_{+}$with $F=$ $\sup \left\{|f(t, 0)|: t \in \mathbb{R}_{+}\right\}$.

(iv) The function $g: \mathbb{R}_{+} \times \mathbb{R}_{+} \times \mathbb{R} \rightarrow \mathbb{R}$ is continuous and there exist functions $a, b: \mathbb{R}_{+} \rightarrow \mathbb{R}_{+}$such that

$$
|g(t, s, x)| \leq a(t) b(s)
$$

for $t, s \in \mathbb{R}_{+}$. Moreover, we assume that

$$
\lim _{t \rightarrow \infty} a(t) \int_{0}^{\beta(t)} b(s) d s=0 .
$$

The main result of [7] is formulated as follows.

Theorem 20. Under the above assumptions, the functional integral equation (75) has at least one solution in the space $B C\left(\mathbb{R}_{+}\right)$.

Notice that (17) is a particular case of (75) with $\alpha(t)=$ $\beta(t)=\gamma(t)=t$.
If we compare assumptions of Theorems 8 and 20, then we see that assumption (b) of Theorem 8 and assumption (ii) of Theorem 20 are essentially distinct.

Next, we prove that assumption (ii) of Theorem 20 is a particular case of assumption (b) of Theorem 8. Indeed, consider the function $\varphi: \mathbb{R}_{+} \rightarrow \mathbb{R}_{+}$defined by $\varphi(t)=$ $M t /(L+t)$ with $0<M<L$. Obviously, $\varphi$ is continuous, $\varphi(0)=$ 0 , and $\varphi$ is nondecreasing (since $\varphi^{\prime}(t)=M L /(L+t)^{2}>0$ ). Since $\varphi^{\prime \prime}(t)=-2 M L /(L+t)^{3}<0, \varphi$ is concave and by Lemma $13, \varphi$ is subadditive. Moreover, since

$$
\beta(t)=\frac{\varphi(t)}{t}=\frac{M}{L+t}<\frac{M}{L}<1,
$$

we have that $\beta \in \mathscr{B}$.

Therefore, we infer that assumption (b) of Theorem 8 is more general than assumption (ii) of Theorem 20. Consequently, Theorem 8 generalizes and improves Theorem 20 (which is the main result of [7]) when $\alpha(t)=\beta(t)=\gamma(t)=t$ for $t \in \mathbb{R}_{+}$.

\section{Conflict of Interests}

The authors declare there is no conflict of interests in the submitted paper.

\section{References}

[1] K. Kuratowski, "Sur les espaces complets," Fundamenta Mathematicae, vol. 15, pp. 301-309, 1930.

[2] G. Darbo, "Punti uniti in trasformazioni a codominio non compatto," Rendiconti del Seminario Matematico della Universitá di Padova, vol. 24, pp. 84-92, 1955.

[3] B. N. Sadovskii, "A fixed-point principle," Functional Analysis and Its Applications, vol. 1, no. 2, pp. 151-153, 1967.

[4] J. Banaś and K. Goebel, Measures of Noncompactness in Banach spaces, vol. 60 of Lecture Notes in Pure and Applied Mathematics, Marcel Dekker, New York, NY, USA, 1980.

[5] R. R. Akhmerov, M. I. Kamenskii, A. S. Potapov, A. E. Rodkina, and B. N. Sadovskii, Measures of Noncompactness and Condensing Operators, vol. 55, Birkhäuser, Basel, Switzerland, 1992.

[6] J. M. Ayerbe Toledano, T. Domínguez Benavides, and G. López Acedo, Measures of Noncompactness in Metric Fixed Point Theory, Birkhäuser, Basel, Switzerland, 1997.

[7] J. Banaś and B. C. Dhage, "Global asymptotic stability of solutions of a functional integral equation," Nonlinear Analysis: Theory, Methods \& Applications, vol. 69, no. 7, pp. 1945-1952, 2008.

[8] J. Banaś and B. Rzepka, "An application of a measure of noncompactness in the study of asymptotic stability", Applied Mathematics Letters, vol. 16, no. 1, pp. 1-6, 2003.

[9] M. A. Geraghty, "On contractive mappings," Proceedings of the American Mathematical Society, vol. 40, pp. 604-608, 1973.

[10] A. Aghajani, J. Banaś, and N. Sabzali, "Some generalizations of Darbo fixed point theorem and applications," Bulletin of the Belgian Mathematical Society. Simon Stevin, vol. 20, no. 2, pp. 345-358, 2013 


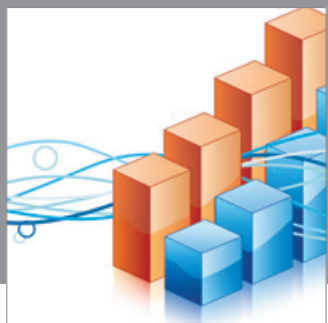

Advances in

Operations Research

mansans

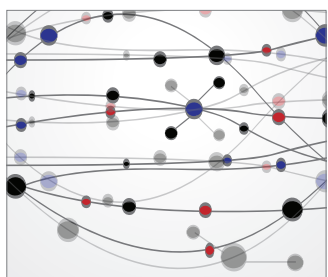

The Scientific World Journal
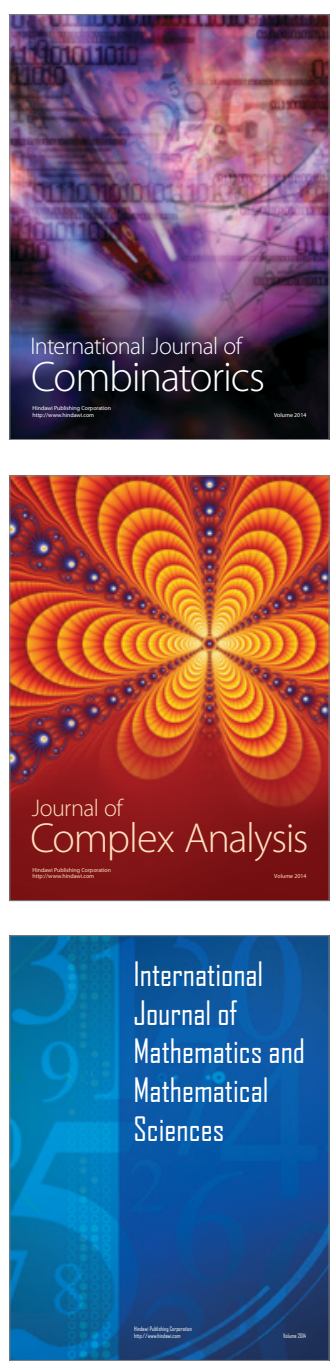
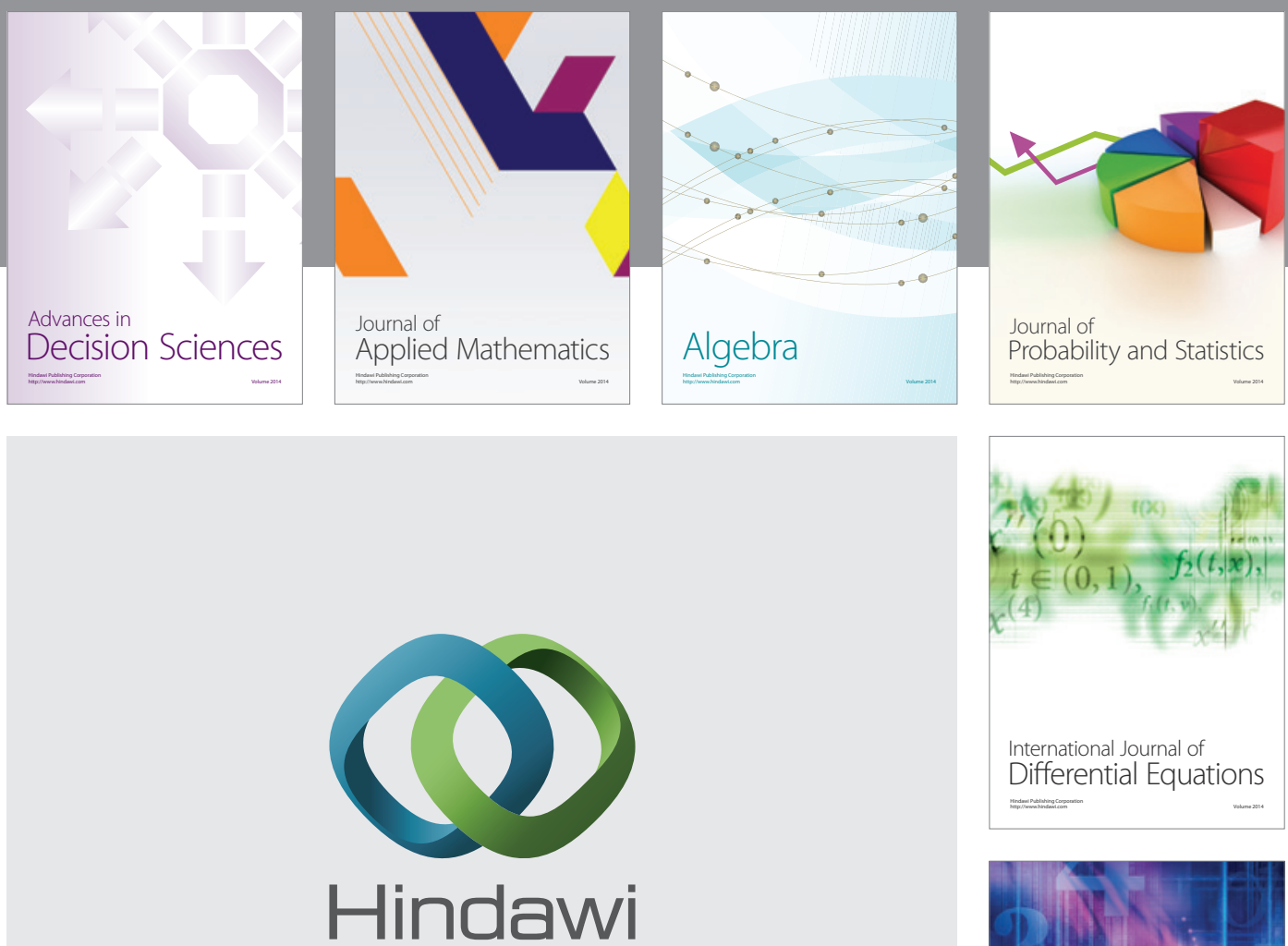

Submit your manuscripts at http://www.hindawi.com
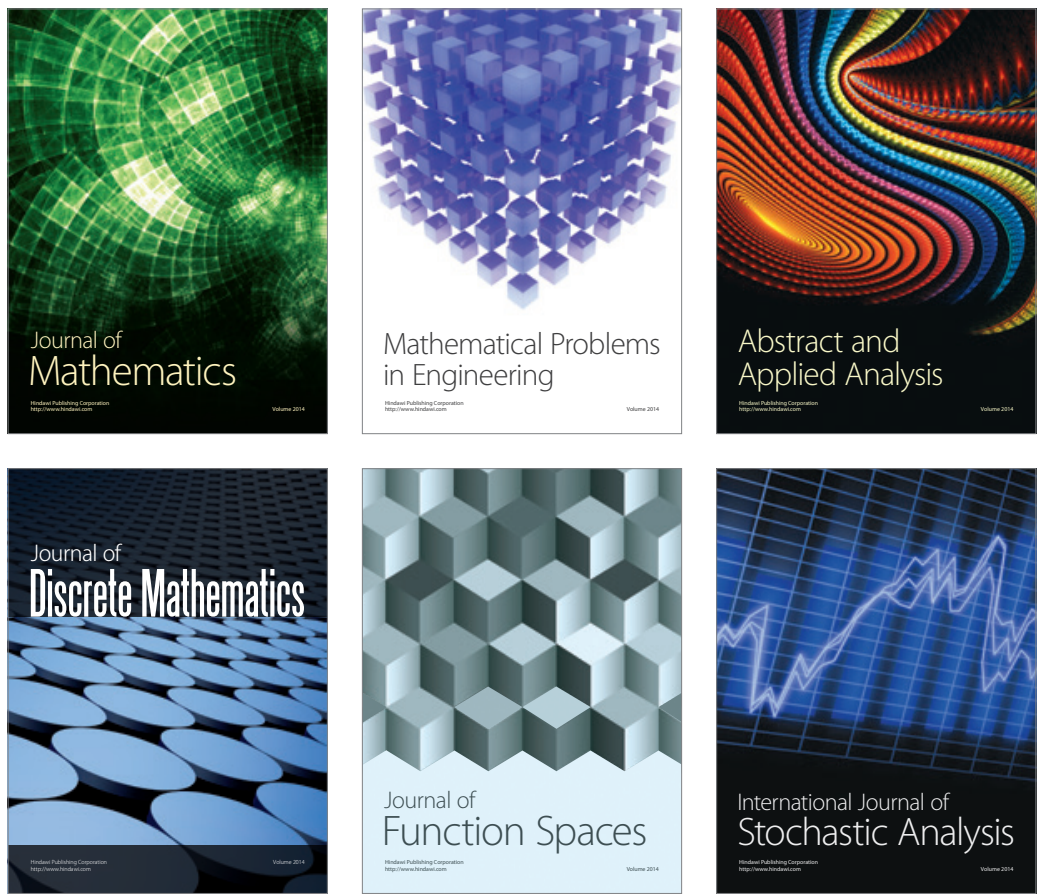

Journal of

Function Spaces

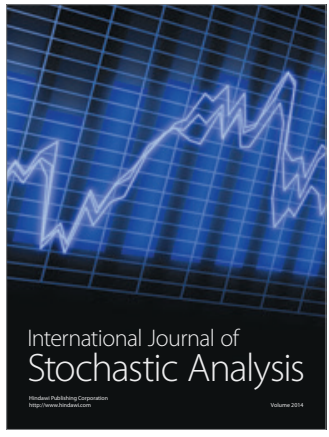

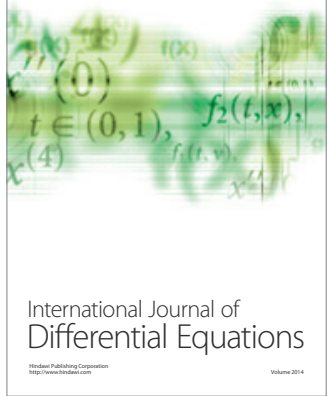
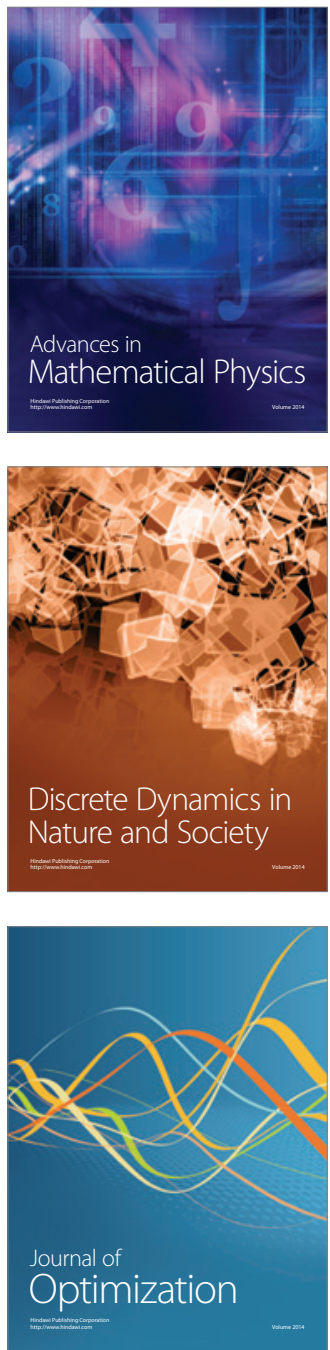